\title{
La página web como herramienta para la retroalimentación en las organizaciones: el caso de las ONGs españolas
}

\section{The website as a tool for feedback in organisations: The case of spanish NGOs}

\author{
Alejandro Tapia Frade. Universidad Europea Miguel de Cervantes \\ Begoña Gómez Nieto. Universidad Europea Miguel de Cervantes \\ Óscar Díaz Chica. Universidad Europea Miguel de Cervantes
}

Recibido: 18-III-2010 - Admitido: 20-IX-2010

\section{Resumen:}

Elementos como imagen pública, transparencia o credibilidad son activos estratégicos de importancia para las organizaciones sin ánimo de lucro (ONGs), y constituyen la base sobre la que contruir su actividad. Por ello, este trabajo analiza la exposición de información y retroalimentación de un centenar de ONGs a través de sus portales web. Se estratificó la muestra según presupuesto, con objeto de determinar diferencias significativas entre los estratos. El método de análisis utilizado fue el análisis de contenido con perspectiva cuantitativa, usando una plantilla con 74 variables. Los resultados ponen de manifiesto que a mayor presupuesto, mayores recursos destinados a la exposición de información. No obstante, a nivel retroalimentativo, se determinó en todos los estratos la ausencia real de mecanismos de retroalimentación para establecer relaciones significativas con los públicos de las ONGs.

Palabras clave:

Comunicación, web 2.0, retroalimentación, visibilidad, portal web, ONGs.

\section{Abstract:}

Elements such as public image, transparency and credibility are important strategic assets for nonprofit organizations (NGOs), and the basis on which to build their business. Therefore, this paper examines the exposure of information and feedback from hundreds of NGOs through their web portals. The sample was stratified according to budget, in order to determine significant differences between the strata. The analytical method used was content analysis with quantitative approach, using a template with 74 variables. The results show that a bigger budget, more resources for information exposure. However, feedback level was determined in all strata of the real absence of feedback mechanisms to establish meaningful relationships with public NGOs.

Keywords:

Communication, web 2.0, feedback, visibility, web portal, NGOs. 


\section{Introducción}

Los proyectos y por tanto la actividad desempeñada por las ONGs dependen en buena medida de las aportaciones económicas de sus públicos. Por tanto, debe considerarse que la imagen es para ellos un activo estratégico fundamental, ya que sin una buena imagen dichas aportaciones se verían fuertemente mermadas. Sin duda, en la construcción de esta imagen uno de los elementos más importantes por ser la principal tarjeta de presentación de las ONGs es la página web. Es uno de los canales fundamentales que utilizan los públicos para entrar en contacto con las organizaciones, y el punto de partida de nuestro estudio.

Conceptos como credibilidad, transparencia o reputación son fundamentales para la viabilidad de las mismas. Por tanto, la exposición de información tanto en lo referente al desarrollo de sus proyectos como en la adecuada gestión de contenidos que trasladan a sus públicos a través de los medios de comunicación son tareas que deberían ser prioritarias en su hacer comunicativo. Además, también es tarea crítica para estas organizaciones escuchar a sus públicos, informarse de las opiniones y demandas de éstos, lo que finalmente deviene en una potente política de retroalimentación comunicativa.

Por otra parte, debemos hacer una diferenciación clara entre dos conceptos muy importantes de la comunicación empresarial: identidad e imagen corporativas. Para Sanz de la Tajada (1994:42-44), la comunicación corporativa consta de tres dimensiones básicas: la primera, permite entrar en un análisis más profundo de lo que la empresa es, y que conforma lo que denomina su "dimensión identidad" -que incluiría su cultura, valores, misión, etc.-; la segunda, representa lo que la empresa dice que es, su "dimensión comunicación", y que proyecta a través de su comunicación, y la tercera determina lo que los públicos creen que es la empresa, su "dimensión imagen", que sería su imagen percibida.

La imagen corporativa es descrita por Van Riel (2000: 27) como el retrato que se tiene de una empresa, es decir cómo ésta es percibida por los públicos objetivo.

Por lo tanto, la identidad es lo que la empresa realmente es y la imagen es la percepción que tienen los distintos públicos objetivos de la empresa, la integración en la mente de sus públicos de todas las señales emitidas por la empresa.

Uno de los canales de obligado uso para todo tipo de organizaciones que quieran estar en contacto con los públicos es Internet a través de su página web. Este hecho implica la creación, mantenimiento, actualización y dinamización de la página si se quiere resultar de interés para los ciudadanos, hecho que podría resultar determinante en la viabilidad de las ONGs. En muchos casos, la inversión se realiza en el momento 
de la creación y puesta en marcha, pero poco a poco el mantenimiento queda en un segundo plano, llegando a convertirse la página en un instrumento que resta en lugar de sumar a la imagen positiva que queremos que tengan los públicos de la organización.

En la presentación ante los distintos públicos mediante este canal, adquieren importancia y son conceptos a tener muy en cuenta.

Tanto la exposición como la retroalimentación de información son entonces tareas fundamentales para la construcción de una adecuada imagen, más aún tras los últimos escándalos que han rodeado estas organizaciones (Diario 20 Minutos, Edición Digital, 09/03/2007 y 03/04/2007), que merman no sólo la imagen de las organizaciones implicadas, sino la de todo el sector (Salvador i Peris, 2000: 5).

Internet constituye un activo estratégico para estas organizaciones (Gálvez Rodríguez, Caba Pérez y López Godoy, 2009:3), apto en términos tanto expositivos como realimentativos, para construir o recuperar la imagen. En términos expositivos, la sala de prensa virtual constituye un potente canal de difusión de noticias y relación con los medios, siendo por tanto un activo fundamental para las organizaciones (Peñalva, 2004: 365). Además, crea sinergias organizativas al sustituir este canal cierta cantidad de recursos necesarios para la realización cotidiana de la tarea periodística. Así, la adecuada gestión del contenido acerca de los proyectos desarrollados por estas organizaciones no es sino una declaración de lo que se hace, y la justificación de en qué se emplean las aportaciones de los públicos a tales tareas.

En términos realimentativos, Internet deviene en un potente canal para averiguar con gran agilidad y rapidez demandas o estados de ánimo de los públicos respecto de las ONGs, y que deberían ser altamente consideradas por éstas, ya que su actividad tiene lugar en buena medida por las aportaciones que sus públicos realizan. De este modo, Internet posibilita la rápida y cercana respuesta de la organización ante sus públicos, que sin duda valorarán positivamente (Vidal, 2004: 311).

Estudios previos similares sobre el desempeño de las ONGs en Internet, como el de Soria Ibáñez (2009) señalan un uso parcial de la potencial retroalimentación informativa del canal Internet, y especialmente de lo que se ha dado en llamar la web 2.0. Pérez y Mateos (2006) mostraron en un estudio sobre ONGs dedicadas a inmigración que en general las webs españolas carecen de utilidades para generar procesos comunicativos entre éstas y sus públicos, hecho también puesto de manifiesto por Ozcelik (2008). Otros trabajos hacen referencia a la falta de uso por parte de las ONGs de este canal en relación a sus stakeholders (Clown y Carter, 1999; Kang y Norton, 2004). 
Acorde con los trabajos anteriormente expuestos, este trabajo recoge los resultados sobre un estudio acerca del uso que las ONGs hacen de sus páginas web, tanto en términos de emisión como de capacidad de retroalimentación de información de las mismas.

\section{Metodología}

Con objeto de investigar en qué medida y cómo comunican las ONGs españolas en sus páginas web, se realizó una plantilla de análisis con 74 variables que ilustran las posibilidades de exposición y retroalimentación comunicativa. Por tanto, el método de análisis elegido fue el análisis de contenido con perspectiva cuantitativa.

La selección de variables utilizadas en la plantilla de análisis junto a la muestra de ONGs usadas en el estudio permite la elaboración de conclusiones fiables, aplicables al conjunto de las mencionadas ONGs. Esto permite presentar una visión cercana al panorama comunicativo que ofrece este tipo de organizaciones.

La plantilla se estructura en primer lugar a dos niveles: valorando la presencia de elementos que posibilitan exposición y retroalimentación informativa ${ }^{1}$. Es decir, si en la página web se articulan mecanismos que posibilitan no sólo la mera exposición de información sino además la posibilidad de intercambio de información para la organización (feedback informativo) y para los públicos (atención personalizada a los distintos públicos).

La exposición de información del sitio web se estructuró en dos sentidos: en primer lugar, se trató la comunicación en cuanto a actividad desempeñada. Adicionalmente se consideró la existencia de un centro o sección destinada específicamente a la relación con sus públicos donde se vuelca contenido -tal como noticias, notas de prensa, boletines, etc.- relacionado con la organización o su actividad en tono periodístico, es decir, la existencia de una sala de prensa virtual, cuyos antecedentes se han situado en los antiguos gabinetes de prensa (Soria Ibañez, 2009b).

Para valorar la comunicación en cuanto a actividad desempeñada, se examinó si existe una sección específica destinada a los proyectos o las tareas realizadas y a qué nivel se encontraba, ordenación y estructura de elementos, etc.

Dicha plantilla ya fue utilizada en otra investigación con propósitos similares (Tapia et al, 2009).

Además, estudios anteriores ya investigaban la dualidad unidireccionalidad - bidireccionalidad comunicativa en un estudio sobre 18 Webs de ONGs (Soria Ibañez, 2009 y 2009b). 
También se valoró si existe una sala de prensa y en qué nivel estructural podía visualizarse. Se examinó la presencia o ausencia de noticias, notas de prensa, recortes de prensa, artículos de investigación, hemeroteca, antigüedad de estos elementos y la facilidad de búsqueda apreciada.

En todos los capítulos antes mencionados se valoró si en la comunicación se utilizaba únicamente texto o si se aprovechaban las cualidades del medio y se incluía fotografía, audio y vídeo.

Por último, para determinar las posibilidades realimentativas de los sitios web se valoró si constaba email, formularios para pedir información y posibilidad de comentar, valorar o escribir sobre la organización y su actividad. Finalmente, se investigó la existencia de nuevas formas de comunicación -vinculadas con la web 2.0 - con altas posibilidades de retroalimentación. Nos referimos a chats, blogs, etc.

Respecto a la ejecución del cuestionario, hay que señalar que en primer lugar se realizó un pretest con una veintena de empresas. Tras realizarlo, se hizo una reunión con objeto de delimitar los errores y corregir el cuestionario. Con el cuestionario resuelto, se hizo otra reunión para tomar una postura común en los tres investigadores responsables respecto a los elementos que ofrecían alguna duda, con el fin de homogeneizar la resolución de problemas.

Posteriormente, la plantilla de análisis definitiva fue ejecutada por los citados investigadores sobre los sitios web de 101 organizaciones sin ánimo de lucro. Dicha recogida de información se efectuó durante el mes de febrero de 2010, en concreto en la primer quincena del citado mes.

A fin de lograr una adecuada representatividad de dichas organizaciones, se seleccionaron dichas organizaciones contando con el presupuesto que habían declarado en sus memorias anuales. Así, se estudiaron organizaciones que ofrecen diferencias notorias a este respecto (en concreto, en un rango que oscila entre los 8.350 euros y los 472 millones de euros, que fueron agrupadas en cuatro grupos: inferior a 100.000 euros, entre 100.001 y 1.000 .000 de euros, entre 1.000 .001 y 10.000.000, y finalmente superior a 10.000.000 de euros ${ }^{2}$ ) teniendo como objetivo ofrecer una visión del sector lo más equilibrada posible. La frecuencia de organizaciones en cada estrato puede apreciarse en la tabla siguiente.

Según los datos ofrecidos por la Fundación Eroski. Dicha fuente ha sido utilizada en otros estudios de análoga temática (Soria Ibañez, 2009: 10). 
Tabla I. Distribución de ONGs analizadas por presupuesto.

\begin{tabular}{|lccc|}
\hline Presupuesto en euros & Frecuencia & Porcentaje & Porcentaje acumulado \\
\hline Inferior a 100.000 & 21 & 20,8 & 20,8 \\
\hline Entre 100.001 y 1.000 .000 & 24 & 23,8 & 44,6 \\
\hline Entre 1.000.001 y 10.000.000 & 38 & 37,6 & 82,2 \\
\hline Superior a 10.000.000 & 18 & 17,8 & 100 \\
\hline Total & 101 & 100 & \\
\hline
\end{tabular}

Se renunció a la proporcionalidad de estratos en este estudio debido a la propia naturaleza del sector: al ser un mercado extremadamente atomizado, con gran número de organizaciones de escaso presupuesto, guardar la debida proporcionalidad implicaría el análisis deficiente de estratos con mayores presupuestos -salvo que se pudiera contar con una muestra muy amplia-. Teniendo en cuenta que uno de los objetivos de este estudio pasa por poner de relevancia diferencias sustanciales en función del presupuesto, y relacionar dichas diferencias con la, a priori, escasa necesidad de presupuesto para gestionar adecuadamente la exposición de estos contenidos, en principio bastante estáticos, se tuvo que renunciar a dicha proporcionalidad.

Antes se ha mencionado que estamos ante un sector extremadamente atomizado. Tan intensa es esta condición, que no se sabe a ciencia cierta cuántas ONGs hay en España (García, 2010, y Galán, 2005). Dicha falta de información se debe a que no existe un registro único en que deban inscribirse. Por su forma jurídica, las ONGs no existen, ya que o son fundaciones o son asociaciones. Sin embargo, no todas las citadas organizaciones son ONGs. Sumadas las de desarrollo según la AECID (Agencia Española de Cooperación Internacional para el Desarrollo) y las de acción social según el Ministerio de Trabajo e Inmigración, serían 1.4403. Sin embargo, Pérez Díaz y López Novo (2003) señalan que contabilizando las ONGs inscritas en los dos agrupaciones mayoritarias, CONGDE (Coordinadora de ONG de Cooperación para el Desarrollo) y la Plataforma de Acción Social, se podrían contabilizar en torno a 3.000 organizaciones, aunque afirman que en total podrían ser en torno a 15.000 .

Para la explotación de datos se utilizó el software SPSS v.15.

\footnotetext{
Según los datos aportados en las páginas web del Ministerio de Trabajo e Inmigración y de la Agencia Española de Cooperación Internacional para el Desarrollo.
} 


\section{Resultados}

\subsection{Introducción}

La primera consideración acerca de la comunicación en Internet entendemos que debe plantearse en términos dicotómicos, es decir, considerar si efectivamente estas organizaciones tienen o no página web. Los resultados, tal y como puede apreciarse en la tabla II, no dejan lugar a dudas: las ONG tienen, en su mayor parte, página web, resultando marginal el porcentaje de dichas organizaciones que no la tienen. Por ello, debemos considerar que se tiene, al menos, la posibilidad de exponer información corporativa en este medio sin distinción en razón de presupuesto, lo cual es compatible, a priori, con la accesibilidad en coste de esta herramienta para todas las organizaciones.

Tabla II. Porcentaje de organizaciones con página web estratificado por presupuesto

\begin{tabular}{|lccc|}
\hline Presupuesto & \multicolumn{2}{c|}{ Tiene Página web } & Total \\
\hline & No & $\mathrm{Si}$ & \\
\hline Inferior a 100.000 & $4,80 \%$ & $95,20 \%$ & $100,00 \%$ \\
\hline Entre 100.001 y 1.000 .000 & $4,20 \%$ & $95,80 \%$ & $100,00 \%$ \\
\hline Entre 1.000 .001 y 10.000 .000 & $2,60 \%$ & $97,40 \%$ & $100,00 \%$ \\
\hline Superior a 10.000 .000 & - & $100,00 \%$ & $100,00 \%$ \\
\hline Total & $3,00 \%$ & $97,00 \%$ & $100,00 \%$ \\
\hline
\end{tabular}

Otra cuestión es el alcance que se desee tener en este medio. Así, la primera consideración de contenido debe ser en qué idioma o idiomas está disponible dicha página. No obstante, la elección dependerá de forma notoria del público al que se desea alcanzar. En el caso de las ONGs, la elección mayoritaria pasa por el empleo exclusivamente del español, si bien puede apreciarse cierta presencia en el uso del español y la lengua regional correspondiente. Finalmente, el uso de más de dos idiomas (usualmente español, la lengua regional e inglés) es también una opción considerada por estas organizaciones. Los resultados, parecidos en todos los niveles presupuestarios, pueden apreciarse en la siguiente tabla. 
Tabla III. Porcentaje de uso de idiomas en página web estratificado por presupuesto

\begin{tabular}{|lccccc|}
\hline Presupuesto & \multicolumn{3}{c|}{ ¿En qué idiomas está disponible? } & Total \\
\hline & $\begin{array}{c}\text { Sólo en } \\
\text { Español }\end{array}$ & $\begin{array}{c}\text { Español e } \\
\text { Inglés }\end{array}$ & $\begin{array}{c}\text { Español } \\
\text { y otra distinta } \\
\text { de inglés }\end{array}$ & $\begin{array}{c}\text { Mas de } \\
\text { dos idiomas }\end{array}$ & \\
\hline Inferior a 100.000 & $89,50 \%$ & & $10,50 \%$ & & $100,00 \%$ \\
\hline Entre 100.001 y 1.000.000 & $55,00 \%$ & $5,00 \%$ & $10,00 \%$ & $30,00 \%$ & $100,00 \%$ \\
\hline Entre 1.000.001 y 10.000.000 & $75,70 \%$ & & $8,10 \%$ & $16,20 \%$ & $100,00 \%$ \\
\hline Superior a 10.000.000 & $72,20 \%$ & $11,10 \%$ & & $16,70 \%$ & $100,00 \%$ \\
\hline Total & $73,40 \%$ & $3,20 \%$ & $7,40 \%$ & $16,00 \%$ & $100,00 \%$ \\
\hline
\end{tabular}

Por tanto, se puede concluir que las ONGs en general tienen presencia en Internet, lo que constituye en sí mismo una declaración de la importancia que dicho canal tiene en el cúmulo de comunicación de estas organizaciones. En ellas, el uso del idioma en las webs debe considerarse en relación al ámbito geográfico objeto de su actividad, lo que podría resultar adecuado si el objeto de su actividad se restringe al territorio nacional, y marginalmente a otros territorios.

\subsection{La exposición de información: proyectos realizados y sala de prensa}

\subsubsection{La exposición de información sobre proyectos realizados}

La primera cuestión relevante acerca de la comunicación sobre la actividad desempeñada es si dicha información tiene presencia en la página web. Además, si ésta se ubica en un lugar específico, y en ese caso a qué nivel estructural se encuentra, parece lógico considerar que si la organización destina un lugar distinto a un nivel estructural importante a esta información de alguna manera reconoce la importancia de este elemento en relación, al menos, con el resto de informaciones disponibles. Los datos obtenidos de la muestra seleccionada ponen de relevancia que en los estratos analizados generalmente se ofrece información acerca de los proyectos que la organización lleva a cabo. Sin embargo, aún siendo mayoría las organizaciones que ofrecen tal información en una sección específica, se advirtieron diferencias relevantes si se toma en consideración el presupuesto de las mismas, tal y como puede apreciarse en la tabla IV. 
Tabla IV. Presencia de una sección específica destinada a la información de proyectos

\begin{tabular}{|lcc|}
\hline & \multicolumn{2}{c|}{ ¿Está la información de proyectos en una sección específica? } \\
\hline Presupuesto (en euros) & No & Si \\
\hline Inferior a 100.000 & $25,00 \%$ & $75,00 \%$ \\
\hline Entre 100.001 y 1.000 .000 & $17,40 \%$ & $82,60 \%$ \\
\hline Entre 1.000 .001 y 10.000 .000 & $5,40 \%$ & $94,60 \%$ \\
\hline Superior a 10.000 .000 & & $100,00 \%$ \\
\hline Global & $10,80 \%$ & $89,20 \%$ \\
\hline
\end{tabular}

Así, se puede afirmar que a mayor presupuesto, mayor probabilidad de existencia de una sección específica destinada a albergar información sobre proyectos -los estadísticos Tau_b de Kendall y Rho de Spearman con valores 0,259 y 0,282 afirman tal relación, con grados de significación en 0,007 y 0,006- . Los títulos más frecuentes para esta sección son Qué hacemos (20,8\% de los casos) y Proyectos (19,8\%). Por otra parte, la mayoría de las webs ofrecen un lugar preeminente en sus webs para esta información -el 96,4\% de ellas la ofrecen a como mucho un clic de distancia-.

\section{La ordenación de la información}

Los datos ponen de manifiesto que la ordenación es frecuente en el caso de ONGs con presupuestos superiores a los 100.000 euros. Todavía más, es más frecuente cuanto mayor es el presupuesto -en este caso, los análisis realizados confirman esta tendencia con un grado de significación de 0,000. Los valores para los estadísticos Tau_b de Kendall y Rho de Spearman fueron de 0,352 y 0,382 respectivamente-. El criterio de ordenación preferido en primer nivel fue el área temática, seguido de la zona geográfica y por último la fecha de realización, sin haberse obtenido diferencias relevantes en función del presupuesto organizativo. Además, usualmente (con porcentajes en torno al 60\% en el cómputo global) junto al contenido textual suelen ofrecerse fotografías de los proyectos, aunque este formato de información tiene mayor presencia en organizaciones con presupuestos mayores (en ONGs con presupuestos superiores a los 10.000.000 de euros, el porcentaje alcanza el $89,9 \%)$.

En conclusión, a mayor presupuesto, mayor probabilidad de disponer de una sección específica destinada a albergar información de proyectos, y también mayor probabilidad de que ésta se encuentre ordenada de forma sistemática. Eso si, en todos los casos en que dicha sección está presente asume una posición estructural importante, que pudiera relacionarse con la alta consideración de este tipo de información en 
relación al resto de contenidos. No en vano, este tipo de información en buena medida justifica las aportaciones de las que son acreedores.

\section{Multimedia en la exposición de proyectos}

A la vista de los resultados, no se puede afirmar que los contenidos sobre proyectos aprovechen las posibilidades que el canal Internet ofrece. La fotografía tiene especial presencia en organizaciones con presupuestos abultados, al igual que el vídeo (de hecho, se pudo confirmar relación estadísticamente significativa y directa entre presencia de vídeo y presupuesto). No obstante, la presencia de audio es en todo caso escasa. Estos hechos tienen especial relevancia, ya que dichos formatos comunicativos ayudarían, bien usados, al desarrollo del discurso organizativo, y además son especialmente aptos para desarrollar discursos basados en la emotividad, tan usados por este tipo de organizaciones.

\subsubsection{La sala de prensa y sus contenidos}

La sala de prensa permite, con gran plasticidad de formatos, la exposición de todo tipo de información a todos los públicos, pero con especial atención a otros difusores de información, los medios de comunicación. La mencionada plasticidad permite la adaptación de contenidos a todo tipo de medios, que pueden acceder a cualquier información de la organización con rapidez, característica ésta fundamental para los medios de comunicación.

Por ello, y al igual que en el caso anterior, la primera consideración pasa por estimar la existencia de este elemento en la página web. Los datos ponen de manifiesto una fuerte división en dos grupos: las organizaciones con presupuestos inferiores al millón de euros (que no suelen incorporar este elemento, con porcentajes de presencia en torno al 35\%) y las que cuentan con presupuestos superiores (que sí suelen incorporarlo, con porcentajes de presencia en torno al 85\%). Huelga decir la importancia estratégica de este elemento. Eso sí, cuando su presencia es un hecho, se le concede gran importancia estructural, ya que el 89,2\% de las webs que tienen esta sección la sitúan a como máximo un clic de distancia respecto de la página de inicio. Los nombre más frecuentes asignados a esta sección son Noticias (22,8\% de los casos) y Sala de Prensa (14,9\%). Además, la sala de prensa suele ser de acceso libre (el 98,5\% de las webs analizadas permitían el libre acceso a dicha sección).

En conclusión, sólo una parte de las ONGs -las de mayor presupuesto-parecen haber entendido la importancia de este elemento. Al igual que en el caso de la comunicación sobre proyectos, cuando se aprecia su presencia, se le concede gran importancia ya que también se sitúa en lugares estructuralmente fundamentales de los sitios web. 


\section{El contenido de la sala de prensa virtual}

En lo referente al contenido de la sala de prensa, en este trabajo se consideró, por un lado, la presencia y periodicidad de noticias, recortes de prensa, artículos de investigación, hemeroteca y newsletter y, por otro, su ubicación considerando las opciones de la sala de prensa, la sección de información corporativa u otra sección distinta de éstas.

En nuestra opinión, éste es un recurso comunicativo de primer orden si lo que se desea es una gestión comunicativa eficiente, especialmente con los medios de comunicación. Su presencia genera importantes sinergias organizativas, ya que libera en buena medida de ciertas tareas a los departamentos de comunicación, que a menudo dedican buena parte del tiempo a la atención a los medios, olvidando la generación de discurso organizativo sostenido a largo plazo -lo que debería ser objetivo primordial-. Además, una adecuada explotación de este recurso podría generar contenidos noticiosos más frecuentes, lo que ayudaría a difundir la actividad de estas organizaciones.

\section{Las noticias}

Los datos obtenidos para la presencia de noticias, expuestos en la tabla V, ponen de manifiesto que las organizaciones de presupuesto inferior no suelen incorporarlas, quizá conscientes de su escasa relevancia ante los medios. Al contrario, las organizaciones con presupuestos elevados las incorporan en la sala de prensa, quizá considerando la misma lógica: al ser organizaciones de carácter benéfico con gran capacidad, se consideran muy expuestas a los medios y a la opinión pública. Dichas noticias suelen estar clasificadas, de acuerdo con los datos analizados, en primer lugar por fecha y después por área temática.

Tabla V. Presencia y ubicación de noticias en web estratificada por presupuesto

\begin{tabular}{|lccccc|}
\hline \multicolumn{5}{c|}{ Presupuesto (en euros) } \\
\hline ¿Contiene? & Inferior a & Entre & Entre & Superior a & Global \\
la web noticias & 100.000 & $100.001 \mathrm{y}$ & $1.000 .001 \mathrm{y}$ & 10.000 .000 & \\
& & 1.000 .000 & 10.000 .000 & \\
\hline No & $46,20 \%$ & $5,00 \%$ & $5,70 \%$ & $10,50 \%$ \\
\hline $\begin{array}{l}\text { Sí, pero está fuera de la Sala de } \\
\text { Prensa y de la información } \\
\text { corporativa }\end{array}$ & $7,70 \%$ & $60,00 \%$ & $5,70 \%$ & $17,40 \%$ \\
\hline Sí, en la PR & & & & \\
\end{tabular}


Sin embargo, de poco vale disponer de una sección específica para el contenido noticioso, e incluso albergar en dicha sección noticias, si ambos no están sometidos a un proceso de revisión y actualización continuo. Los datos, estratificados por presupuesto y mostrados en la tabla VI, ponen de relevancia que las organizaciones de menor prepuesto no sólo tienen en menor medida una sección específica en la web y noticias, sino que además cuando existen ambos elementos, el proceso de actualización de las noticias es bastante amplio. Las de mayor presupuesto, al contrario, actualizan sus noticias constantemente y cada poco tiempo. Este hecho es sin duda compatible con la realidad, pues el proceso de revisión y actualización de noticias es laborioso, y suele exigir la dedicación de ciertos recursos humanos, circunstancia que no todas las ONGs pueden presupuestariamente asumir.

De hecho, el análisis estadístico complementario realizado permite afirmar que cuanto mayor es el presupuesto, menor es el tiempo medio de actualización de las noticias -los coeficientes de correlación Tau_b de Kendall y Rho de Spearman, con valores -0,426 y -0,486 y grado de significación para ambos en 0,000 sostienen tal argumento-.

Tabla VI. Tiempo de actualización de noticias estratificada por presupuesto

\begin{tabular}{|lccccr|}
\hline & \multicolumn{5}{c|}{ Presupuesto (en euros) } \\
\hline $\begin{array}{l}\text { ¿Cada cuánto tiempo se } \\
\text { actualizan las noticias? }\end{array}$ & $\begin{array}{c}\text { Inferior a } \\
100.000\end{array}$ & $\begin{array}{c}\text { Entre 100.001 } \\
\text { y } 1.000 .000\end{array}$ & $\begin{array}{c}\text { Entre 1.000.001 } \\
\text { y } 10.000 .000\end{array}$ & $\begin{array}{c}\text { Superior a } \\
10.000 .000\end{array}$ & Global \\
\hline Todos los días o continuamente & & $13,30 \%$ & $10,70 \%$ & $50,00 \%$ & $20,90 \%$ \\
\hline $\begin{array}{l}\text { Varias veces a la semana, } \\
\text { de forma constante }\end{array}$ & $16,70 \%$ & $6,70 \%$ & $28,60 \%$ & $27,80 \%$ & $22,40 \%$ \\
\hline Una vez a la semana & & & $10,70 \%$ & $5,60 \%$ & $6,00 \%$ \\
\hline Más de una vez a la semana & $33,30 \%$ & $66,70 \%$ & $35,70 \%$ & $11,10 \%$ & $35,80 \%$ \\
\hline No se actualiza el contenido & $50,00 \%$ & $13,30 \%$ & $14,30 \%$ & $5,60 \%$ & $14,90 \%$ \\
\hline
\end{tabular}

Entendemos que la actualización de contenidos como noticias y notas de prensa debiera en principio ser constante, aunque implica la disponibilidad de recursos que por otra parte no están al alcance de todas las organizaciones. Es en este punto donde se aprecia la consideración de disponibilidad de recursos antes descrita, ya que se ha podido demostrar que cuanto mayor es el presupuesto, menor es el tiempo medio de actualización de noticias. 


\section{Los recortes de prensa}

Los datos referentes al siguiente elemento considerado en este análisis, los recortes de prensa, muestran en general un pobre nivel de presencia, tal y como queda señalado en la tabla siguiente. Si bien se aprecia mayor presencia y mejor ubicación en las organizaciones de mayor presupuesto, la misma sólo puede calificarse de deficiente en todas las organizaciones consideradas.

Tabla VII. Presencia y ubicación de recortes de prensa en web estratificada por presupuesto

\begin{tabular}{|lccccc|}
\hline \multicolumn{5}{|c|}{ Presupuesto (en euros) } \\
\hline $\begin{array}{l}\text { ¿Existen recortes de } \\
\text { prensa en la web? }\end{array}$ & $\begin{array}{c}\text { Inferior a } \\
100.000\end{array}$ & $\begin{array}{c}\text { Entre 100.001 } \\
\text { y } 1.000 .000\end{array}$ & $\begin{array}{c}\text { Entre 1.000.001 } \\
\text { y } 10.000 .000\end{array}$ & $\begin{array}{c}\text { Superior a } \\
10.000 .000\end{array}$ & Global \\
\hline No & $91,70 \%$ & $100,00 \%$ & $82,40 \%$ & $66,70 \%$ & $84,50 \%$ \\
\hline $\begin{array}{l}\text { Sí, pero está fuera de la } \\
\text { Sala de Prensa y de la } \\
\text { información corporativa }\end{array}$ & $8,30 \%$ & & & & $1,20 \%$ \\
\hline Sí, en la PR & & & $17,60 \%$ & $33,30 \%$ & $14,30 \%$ \\
\hline
\end{tabular}

\section{Artículos de investigación}

En cuanto a la existencia de artículos de investigación y reportajes el estudio muestra un comportamiento diferente en función del presupuesto de la organización. Los datos, expuestos en la tabla VIII, permiten sugerir que la presencia de este elemento es mayor en organizaciones con presupuesto superior, y menor en caso contrario. También en este caso puede considerarse compatible con la realidad, puesto que al igual que en el caso de las noticias, la elaboración de artículos de investigación y reportajes extensos necesita de ciertos recursos no siempre alcanzables por estas organizaciones. No obstante, los análisis complementarios realizados no permiten afirmar relación directa estadísticamente significativa entre presencia, ubicación y presupuesto organizativo. 
Tabla VIII. Presencia y ubicación de artículos de investigación y reportajes en web.

\begin{tabular}{|lccccc|}
\hline \multicolumn{5}{c|}{ Presupuesto (en euros) } \\
\hline $\begin{array}{l}\text { ¿Existen artículos de } \\
\text { investigación y } \\
\text { reportajes en la web? }\end{array}$ & $\begin{array}{c}\text { Inferior a } \\
100.000\end{array}$ & $\begin{array}{c}\text { Entre 100.001 } \\
\text { y } 1.000 .000\end{array}$ & $\begin{array}{c}\text { Entre 1.000.001 } \\
\text { y } 10.000 .000\end{array}$ & $\begin{array}{c}\text { Superior a } \\
10.000 .000\end{array}$ & Global \\
\hline No & $69,20 \%$ & $34,80 \%$ & $48,60 \%$ & $29,40 \%$ & $44,30 \%$ \\
\hline $\begin{array}{l}\text { Sí, pero está fuera de la } \\
\text { Sala de Prensa y de la } \\
\text { información corporativa }\end{array}$ & $30,80 \%$ & $52,20 \%$ & $45,70 \%$ & $23,50 \%$ & $40,90 \%$ \\
\hline Sí, en la PR & & & & & \\
\hline
\end{tabular}

Por tanto, la gestión de contenidos como artículos de investigación y reportajes muestra un comportamiento variable, con menor presencia en organizaciones de menor presupuesto, lo que en principio podría relacionarse con su limitada disponibilidad de recursos antes enunciada.

\section{La hemeroteca}

El siguiente elemento considerado, la presencia y ubicación de hemerotecas con contenidos antiguos, tiene una consideración esencialmente estructural, ya que hace referencia al modo de almacenamiento de contenido noticioso. Los datos muestran un escaso porcentaje de aplicación en las páginas web independientemente del presupuesto organizativo. Los datos concretos de presencia y ubicación pueden verse en la tabla a continuación mostrada.

No obstante, cuando su improbable presencia es un hecho, se suele actualizar su contenido anualmente (69,2\% de los casos) y la antigüedad del mismo suele ser de entre 5 y 10 años (42,9\% de los casos). 
Tabla IX. Presencia y ubicación de hemeroteca en web.

\begin{tabular}{|lccccc|}
\hline \multicolumn{1}{|c|}{ Presupuesto (en euros) } \\
\hline $\begin{array}{l}\text { ¿Existe una Hemeroteca con } \\
\text { contenido antiguo en la web? }\end{array}$ & $\begin{array}{c}\text { Inferior a } \\
100.000\end{array}$ & $\begin{array}{c}\text { Entre 100.001 } \\
\text { y 1.000.000 }\end{array}$ & $\begin{array}{c}\text { Entre 1.000.001 } \\
\text { y } 10.000 .000\end{array}$ & $\begin{array}{c}\text { Superior a } \\
10.000 .000\end{array}$ & Global \\
\hline No & $95,00 \%$ & $87,00 \%$ & $81,10 \%$ & $82,40 \%$ & $85,60 \%$ \\
\hline $\begin{array}{l}\text { Sí, pero está fuera de la Sala } \\
\text { de Prensa y de la información } \\
\text { corporativa }\end{array}$ & $5,00 \%$ & $4,30 \%$ & $2,70 \%$ & $5,90 \%$ & $4,10 \%$ \\
\hline Sí, en la PR & & & & & \\
\hline
\end{tabular}

En conclusión, la presencia de hemerotecas es residual, aunque en los contados casos en que pudo constatarse su presencia la gestión era adecuada -con actualizaciones anuales de este recurso, y albergando contenidos de cierta antigüedad-.

\section{El boletín periódico o newsletter}

Otra herramienta vehicular de difusión interesante es el newsletter o boletín periódico. Los datos expuestos en la tabla siguiente ponen de manifiesto que la presencia es mayor cuanto mayor presupuesto organizativo disponible (las correlaciones Tau_b de Kendall y Rho de Spearman, con valores 0,249 y 0,284, en ambos casos con grado de significación en 0,005, sostienen tal hecho).

No obstante, también parece relevante señalar su ubicación mayoritaria, independientemente del presupuesto considerado, en una sección distinta de la sala de prensa y la información corporativa. Dicho boletín suele ser accesible sin suscripción (70,6\% de los casos sin estratificar) y su acceso más frecuente es mediante descarga (61,7\% de los casos sin estratificar por presupuesto). Finalmente, su periodicidad más frecuente es mensual (36,6\% del total de casos) o mayor $(53,7 \%)$. 
Tabla X. Presencia y ubicación de newsletter en web.

\begin{tabular}{|lccccr|}
\hline & \multicolumn{5}{c|}{ Presupuesto (en euros) } \\
\hline $\begin{array}{l}\text { Newsletters o Boletines } \\
\text { Periódicos }\end{array}$ & $\begin{array}{c}\text { Inferior } \\
\text { a 100.000 }\end{array}$ & $\begin{array}{c}\text { Entre 100.001 } \\
\text { y } 1.000 .000\end{array}$ & $\begin{array}{c}\text { Entre 1.000.001 } \\
\text { y 10.000.000 }\end{array}$ & $\begin{array}{c}\text { Superior a } \\
10.000 .000\end{array}$ & Global \\
\hline No & $75,00 \%$ & $47,80 \%$ & $51,40 \%$ & $11,10 \%$ & $48,00 \%$ \\
\hline $\begin{array}{l}\text { Sí, pero está fuera de la Sala } \\
\text { de Prensa y de la información } \\
\text { corporativa }\end{array}$ & $25,00 \%$ & $43,50 \%$ & $35,10 \%$ & $55,60 \%$ & $38,80 \%$ \\
\hline $\begin{array}{l}\text { Sí, fuera de la Sala de Prensa } \\
\text { y dentro de una sección de } \\
\text { Información corporativa }\end{array}$ & & & & & \\
\hline Sí, en la Sala de Prensa & & & & & \\
\hline
\end{tabular}

En nuestra opinión, la presencia de newsletters o boletines periódicos es también una cuestión notable, especialmente si no se dispone de recursos noticiosos, ya que en caso de dicha ausencia el boletín constituye uno de los pocos recursos con que poder dar cuenta periódica de su actividad. El tono general en este caso no difiere en demasía de lo mencionado respecto del resto de recursos informativos: de hecho también se pudo determinar relación estadísticamente significativa entre este elemento y el presupuesto.

\section{Multimedia en la sala de prensa}

En este estudio se consideró la presencia de audio y vídeo en la sala de prensa. En lo que respecta al audio, hay que señalar la presencia residual de este elemento en todos los estratos analizados. Además, cuando tiene presencia, la locución se realiza en un único idioma, limitando por tanto el uso de dicho recurso a una comunidad reducida. Por tanto, debemos afirmar que en el caso de los archivos de audio no se aprovecha el potencial de Internet para ofrecer formatos alternativos al texto y la imagen que bien pudieran ser aprovechados por ciertos medios de comunicación, y llegar por tanto a la audiencia que recurre a tales medios para informarse. Los datos concretos pueden apreciarse en la tabla siguiente. 
Tabla XI. Presencia y ubicación de audio y vídeo en web.

\begin{tabular}{|c|c|c|c|c|c|c|}
\hline \multirow{2}{*}{\multicolumn{2}{|c|}{$\begin{array}{l}\text { Presencia y ubicación de audio y } \\
\text { vídeo en web }\end{array}$}} & \multicolumn{5}{|c|}{ Presupuesto (en euros) } \\
\hline & & $\begin{array}{c}\text { Inferior a } \\
100.000\end{array}$ & $\begin{array}{c}\text { Entre } 100.001 \\
\text { y } 1.000 .000\end{array}$ & $\begin{array}{c}\text { Entre } 1.000 .001 \\
\text { y } 10.000 .000\end{array}$ & $\begin{array}{l}\text { Superior a } \\
10.000 .000\end{array}$ & Global \\
\hline \multirow{4}{*}{$\begin{array}{l}\text { ¿Hay vídeos en } \\
\text { la web? }\end{array}$} & No & $60,00 \%$ & $82,60 \%$ & $51,40 \%$ & $27,80 \%$ & $56,10 \%$ \\
\hline & $\begin{array}{l}\text { Sí, en la Sala } \\
\text { de Prensa }\end{array}$ & & $4,30 \%$ & $10,80 \%$ & $5,60 \%$ & $6,10 \%$ \\
\hline & $\begin{array}{l}\text { Sí, dentro de una } \\
\text { sección de infor- } \\
\text { mación corporativa }\end{array}$ & & & $2,70 \%$ & $16,70 \%$ & $4,10 \%$ \\
\hline & $\begin{array}{l}\text { Sí, pero está fuera } \\
\text { de ambas }\end{array}$ & $40,00 \%$ & $13,00 \%$ & $35,10 \%$ & $50,00 \%$ & $33,70 \%$ \\
\hline \multirow{3}{*}{$\begin{array}{l}\text { ¿Hay archivos } \\
\text { de audio? }\end{array}$} & No & $90,00 \%$ & $95,70 \%$ & $88,90 \%$ & $94,40 \%$ & $91,80 \%$ \\
\hline & $\begin{array}{l}\text { Sí, en la Sala } \\
\text { de Prensa }\end{array}$ & & & $5,60 \%$ & & $2,10 \%$ \\
\hline & $\begin{array}{l}\text { Sí, pero está fuera } \\
\text { de la Sala de Prensa } \\
\text { y la Información } \\
\text { corporativa }\end{array}$ & $10,00 \%$ & $4,30 \%$ & $5,60 \%$ & $5,60 \%$ & $6,20 \%$ \\
\hline
\end{tabular}

La presencia de archivos de vídeo muestra un comportamiento menos uniforme que el antes descrito. Si bien se usa en mayor medida por organizaciones potentes (con presupuesto superior a los 10 millones), en el resto de estratos no muestra un comportamiento uniforme. En cualquier caso, hay que señalar su ubicación usualmente fuera de la sección de sala de prensa e información corporativa. En el caso de existir tales vídeos en página web, éstos van sólo parcialmente provistos de descripciones (concretamente, en el $45,5 \%$ de los casos analizados con presupuestos inferiores a 100.000 euros, un $75 \%$ en el caso de organizaciones de entre 100.000 y 1.000 .000 de euros, un 64,7\% en organizaciones de entre 1 y 10 millones de euros, y un 84,6\% en el caso de ONGs con presupuestos superiores a 10 millones), lo que constituye una evidente falta de atención que debería ser corregida. Las pruebas estadísticas complementarias realizadas 
permiten afirmar relación objetiva estadísticamente significativa entre presupuesto y presencia de vídeo, pero no de audio .

\section{Los buscadores de información}

Sin duda, un aspecto fundamental en la página web es la facilidad de búsqueda de sus distintos contenidos. La potencialmente elevada cantidad de información en web no sólo requiere una adecuada estructuración de la misma, sino también la disposición de herramientas que simplifiquen la búsqueda de cierta información entre todas las restantes. Este trabajo consideró la existencia de dichas herramientas de búsqueda a dos posiciones: a nivel de contenido general, y a nivel específico de noticias, hemeroteca, artículos de investigación y recortes de prensa. Si bien los datos manifiestan un uso muy escaso de este recurso en el ámbito de las noticias (86,2\% de ausencia considerando todos los casos), si se considera dicho buscador a nivel general de web, queda confirmado que a mayor presupuesto, mayor probabilidad de presencia de este elemento -los coeficientes de correlación Tau_b de Kendall y Rho de Spearman, con valores 0,208 y 0,227 y grado de significación en 0,030 y 0,029 respectivamente sostienen tal argumento-, aunque en todo caso estamos ante un elemento cuya presencia es manifiestamente mejorable, ya que el porcentaje de presencia general es apenas del $28 \%$.

\subsection{La retroalimentación de información: la web 2.0 y otras formas de comunicación.}

La retroalimentación de información es sin duda una cuestión básica para cualquier organización que pretenda operar en el mercado de forma mínimamente eficiente, ya que sin dicha retroalimentación no sería posible atender las demandas de los mercados, cada vez más dinámicos. Además, la adopción de un modelo simétrico de relación entre una organización y sus públicos requiere de dicha bidireccionalidad en la comunicación.

Internet es un canal potente para este tipo de acciones, ya que permite disponer de información en tiempo real sobre muy diversas cuestiones de mercado. Todavía más, con la llegada de la web 2.0 esas posibilidades de retroalimentación informativa se ven sustancialmente incrementadas, hasta el punto de la construcción cooperativa de discurso en medios digitales.

\footnotetext{
4 En el caso de vídeo y presupuesto, los indicadores de correlaciones Tau_b de Kendall -con valor 0,211- y Rho de Spearman -con valor 0,232-, y las pruebas de significación bilateral con valores 0,019 y 0,022 permiten afirmar la misma con nivel crítico menor que 0,05, pero no a nivel 0,01. En el caso de audio y presupuesto, las pruebas de significación con valores 0,929 y 0,928 eliminan la posibilidad de establecer relación objetiva. Además, las correlaciones con valores -0,008 y -0,009 nos acercan a la consideración de independencia de estas variables.
} 
En efecto, Internet constituye un medio en el que las organizaciones están cada vez más expuestas a las opiniones de consumidores y otros públicos. Esta exposición a lo público requiere mayores dosis de transparencia informativa, elementos estos particularmente importantes en las ONGs, que dependen en buena medida de factores como la transparencia o la credibilidad si lo que pretenden es la captación de fondos para sus operaciones.

Por ello esta investigación tuvo muy en cuenta el estudio de elementos tradicionales de retroalimentación comunicativa como los formularios en web, emails o teléfonos y otros típicos de la web 2.0 como los blogs, la posibilidad de valorar o comentar noticias u opiniones sobre los proyectos, los chats, etc.

Respecto a los elementos tradicionales en web, emails y teléfonos son elementos presentes de forma generalizada en todas las organizaciones (87,8\% de presencia de teléfono y $89,9 \%$ en el caso del email) sin haberse encontrado diferencias significativas considerando el presupuesto de las mismas. No obstante, los números y emails facilitados remiten a buzones genéricos (98,8\% de los teléfonos y 87,5\% de los emails encontrados sobre el cómputo global) en todos los niveles presupuestarios analizados.

Sin embargo, no sucede lo mismo en el caso de formularios de reclamaciones y contacto, cuya presencia es mayoritaria únicamente en organizaciones de mayor capacidad presupuestaria. Dichos formularios son herramientas que proporcionan retroalimentación informativa inmediata y pueden servir para recabar información en poco tiempo sobre cierto hecho. Además, no tienen un mantenimiento costoso, por lo que sería recomendable su implementación en todo tipo de organizaciones, independientemente de su capacidad presupuestaria.

La posibilidad de comentar o valorar noticias no ofrece mejores perspectivas. Los datos ponen de manifiesto la inexistencia sistemática de estos elementos, salvo de forma residual en las organizaciones más potentes (con apenas el 5,6\% de presencia en ese estrato), lo que constituye una evidente falta de atención a medios y público en general, no permitiendo por tanto el diálogo en esta parcela. En el caso de los comentarios y valoraciones sobre los proyectos desarrollados, la situación es parecida. Si bien la posibilidad de comentar los proyectos desarrollados es algo mayor, sigue siendo residual (concretamente, con un porcentaje de presencia del 3,3\% en global), sin apreciarse diferencias significativas en función del presupuesto asumido. Respecto de la valoración de los proyectos, su presencia se reduce al 1,1\% de las webs estudiadas. Teniendo en cuenta la facilidad y el bajo coste de implementación de estos sistemas, las ONGs deberían implementarlo con independencia del presupuesto, pues permite el diálogo, elemento indispensable si se pretende establecer una relación simétrica entre estas organizaciones y sus públicos. 
Los datos analizados para nuevas formas de comunicación ofrecen una visión más optimista de estas organizaciones, aunque a nuestro parecer insuficiente, tal y como puede apreciarse en la tabla XII.

Tabla XII. Presencia de nuevas formas de comunicación estratificado por presupuesto.

\begin{tabular}{|c|c|c|c|c|c|c|}
\hline \multicolumn{2}{|c|}{ Nuevas formas de comunicación } & \multicolumn{5}{|c|}{ Presupuesto (en euros) } \\
\hline & & $\begin{array}{c}\text { Inferior a } \\
100.000\end{array}$ & $\begin{array}{c}\text { Entre } 100.001 \\
\text { y } 1.000 .000\end{array}$ & $\begin{array}{c}\text { Entre } 1.000 .001 \\
\mathrm{y} 10.000 .000\end{array}$ & $\begin{array}{l}\text { Superior a } \\
10.000 .000\end{array}$ & Global \\
\hline $\begin{array}{l}\text { Servicio de alertas } \\
\text { de noticias por } \\
\text { móvil } \\
\\
\text { y } \\
\text { y }\end{array}$ & $\begin{array}{c}\text { No } \\
\text { Sí, en la Sala } \\
\text { de Prensa } \\
\text { Sí, pero está fuera } \\
\text { de la Sala de prensa } \\
\text { y de la Información } \\
\text { corporativa }\end{array}$ & $100,00 \%$ & $100,00 \%$ & $97,10 \%$ & $88,90 \%$ & $2,20 \%$ \\
\hline $\begin{array}{l}\text { Servicio de } \\
\text { noticias por RSS }\end{array}$ & $\begin{array}{c}\text { No } \\
\text { Sí, en la Sala } \\
\text { de Prensa } \\
\text { Sí, pero está fuera } \\
\text { de la Sala de prensa } \\
\text { y de la Información } \\
\text { corporativa }\end{array}$ & $16,70 \%$ & $\begin{array}{c}68,20 \% \\
9,10 \%\end{array}$ & $27,80 \%$ & $\begin{array}{c}55,60 \% \\
5,60 \%\end{array}$ & $\begin{array}{c}70,20 \% \\
3,20 \%\end{array}$ \\
\hline $\begin{array}{l}\text { Presencia en las } \\
\text { redes sociales }\end{array}$ & $\begin{array}{l}\text { No } \\
\text { Sí }\end{array}$ & $\begin{array}{l}63,20 \% \\
36,80 \%\end{array}$ & $\begin{array}{l}65,20 \% \\
34,80 \%\end{array}$ & $\begin{array}{l}78,40 \% \\
21,60 \%\end{array}$ & $\begin{array}{l}50,00 \% \\
50,00 \%\end{array}$ & $\begin{array}{l}67,00 \% \\
33,00 \%\end{array}$ \\
\hline
\end{tabular}

En cuanto a la implementación de un sistema de alertas de noticias por móvil, la situación no puede calificarse de buena, ya que únicamente las organizaciones de mayor presupuesto presentan como opción, aunque ampliamente poco frecuente, este sistema.

El sistema de suscripción a un servicio de noticias por RSS es una opción más frecuentemente acogida, tal y como puede apreciarse en la tabla superior. Si bien, en línea con lo antes expuesto, es una opción más frecuente en el caso de organizaciones más potentes, el análisis complementario realizado no permite afirmar 
relación directa estadísticamente significativa entre presupuesto y probabilidad de presencia de este sistema de distribución de noticias.

La situación antes descrita se repite para el caso de las redes sociales, si bien la presencia de este elemento es más frecuente en todos los estratos analizados, llegando a la mitad de los casos de organizaciones con presupuestos altos. En este caso tampoco se encontró relación de dependencia estadísticamente significativa entre presupuesto y presencia. En cualquier caso, y para todos los estratos, la red social preferida es Facebook, si bien hay que considerar la alta volatilidad de estas herramientas.

Los blogs son herramientas fundamentales en la web 2.0. El porcentaje de presencia de este elemento es el $30,4 \%$ considerando todas las organizaciones, sin deferencias importantes al considerar el presupuesto organizativo. Cuando dicho elemento está presente, la situación más frecuente es que se pueda escribir comentarios $(70 \%$ de los casos analizados, sin haberse podido establecer relación estadísticamente significativa ${ }^{5}$ entre presupuesto y escritura de comentarios), pero no se permite ni escribir noticias ni aún menos la publicación directa de las mismas. Es decir, en tanto que se permite el comentario se inicia un diálogo, pero dado que no se permite la escritura ni la publicación directa de noticias no se puede hablar de cogeneración de discurso, sino a lo sumo de matización del mismo.

Por tanto, debemos señalar que en el caso de estas organizaciones se desaprovecha el potencial comunicativo de estas herramientas, siendo en nuestra opinión un error de amplitud. Similar consideración ha de extenderse para el caso de blogs, que si bien su presencia es más frecuente, sigue siendo ampliamente insuficiente. Por ello, aunque se acredite cierta bidireccionalidad en el improbable caso de presencia de blogs, no se puede hablar de simetría de relación entre público y organización, ya que la relación entre ONG y público está ampliamente limitada por parte del primero.

Finalmente, hay que mencionar en qué medida se ofrece la posibilidad de realizar donaciones en línea, bien a proyectos específicos o con carácter genérico, y la posibilidad de hacerse socio de las ONGs. Los datos, expuestos en la tabla XIII, manifiestan un porcentaje residual en la posibilidad de donar únicamente para proyectos específicos, siendo mucho más frecuente la posibilidad de hacer donaciones genéricas a la organización.

Dicha relación se intentó acreditar mediante el uso de correlaciones Tau_b de Kendally Rho de Spearman, resultando los coeficientes de correlación muy bajos y los grados de significación muy altos. 
Tabla XIII. Aportaciones a través de página web. Existencia.

\begin{tabular}{|lccccc|}
\hline \multicolumn{7}{|c|}{ Presupuesto (en euros) } & & & \\
\hline $\begin{array}{l}\text { ¿Es posible aportar } \\
\text { dinero a través de la web? }\end{array}$ & $\begin{array}{c}\text { Inferior a } \\
100.000\end{array}$ & $\begin{array}{c}\text { Entre } 100.001 \\
\text { y } 1.000 .000\end{array}$ & $\begin{array}{c}\text { Entre } 1.000 .001 \\
\text { y } 10.000 .000\end{array}$ & $\begin{array}{c}\text { Superior } \\
\text { a } 10.000 .000\end{array}$ & Global \\
\hline Donaciones a proyectos especificos & $10,00 \%$ & & & & $2,10 \%$ \\
\hline Donaciones genericas a la ONG & $10,00 \%$ & $54,50 \%$ & $27,00 \%$ & $61,10 \%$ & $36,10 \%$ \\
\hline Ambos & $5,00 \%$ & & $10,80 \%$ & $11,10 \%$ & $7,20 \%$ \\
\hline Ninguno & $75,00 \%$ & $45,50 \%$ & $62,20 \%$ & $27,80 \%$ & $54,60 \%$ \\
\hline
\end{tabular}

Así, si bien, y como consecuencia de lo anterior, no parece interesar a las ONGs la opinión de sus públicos, si manifiestan cierto interés por las aportaciones que éstos realizan. Tanto es así, que la mayoría de las ONGs estudiadas posibilitan la admisión de socios via web, y una parte importante las donaciones genéricas a la organización.

Respecto a la última cuestión, la posibilidad de hacerse socio a través de la web, los resultados indican, sin grandes variaciones en razón de presupuesto cuando éste es superior a 100.000 euros, que la mayoría de las organizaciones incorporan esta posibilidad en sus webs (66,30\% en términos globales). Para las ONGs con presupuesto inferior a 100.000 euros, el porcentaje baja al 55\%.

\section{Conclusiones}

En relación con la exposición de información, habría que señalar que si bien la mera presencia en Internet está resuelta de forma positiva en todos los estratos analizados, puede afirmarse con carácter general que cuanto mayor es el presupuesto organizativo, mayor disponibilidad de elementos comunicativos de tipo expositivo.

Así, respecto a la comunicación de actividad desempeñada puede concluirse cierta relación entre presupuesto organizativo y presencia de una sección específica de comunicación de la actividad desempeñada. Resulta interesante esta cuestión, ya que la presencia de una sección específica a un nivel estructural importante señala de alguna manera la importancia concedida a este elemento en relación al resto. Por tanto, debe sugerirse que cuanto mayor es el presupuesto, mayor conciencia de la importancia que la comunicación de los proyectos realizados o a realizar tiene en relación al resto de informaciones que se presenta. 
No obstante, no puede decirse lo mismo sobre la explotación de posibilidades que Internet ofrece para este tipo de información. La presencia de vídeo y audio es escasa, aunque debieran utilizarse con intensidad, ya que estos formatos son especialmente aptos para la elaboración de discursos basados en la emotividad, fundamentales para este tipo de organizaciones.

La sala de prensa virtual y sus contenidos ofrecen una importante dependencia del presupuesto de la organización. Podemos concluir que cuanto mayor es el presupuesto, más presencia de este elemento, y más contenido en éste se alberga.

En concreto, cuanto mayor es el presupuesto, mayor probabilidad de presencia de noticias, notas de prensa y boletines de información, mayor probabilidad de actualización de estos elementos en menor tiempo y mayor probabilidad de presencia de buscadores. Sin embargo, hay que considerar en este punto la disponibilidad de recursos de organizaciones de escaso presupuesto, en principio menor, que podría explicar su falta de uso de estos elementos.

En relación con los mecanismos de retroalimentación comunicativa que Internet ofrece, podemos señalar que la mayoría de las organizaciones analizadas desaprovechan el potencial de este medio y no ofrecen posibilidades reales de retroalimentación con independencia del presupuesto organizativo, ignorando por tanto la opinión de sus públicos en todos los estratos analizados.

En concreto, si bien la presencia de emails y teléfonos de contacto es frecuente, éstos usualmente remiten a buzones genéricos de información. Además, la presencia de elementos de la web 2.0, que facilitan la retroalimentación de información, es prácticamente inexistente en todos los casos. No se implementan Blogs, servicios de alerta por móvil, sindicación de noticias por RSS, sistemas de relación en redes sociales ni se permite comentar noticias en la web.

Sorprendentemente, la mayoría de ONGs admiten donaciones y permiten la admisión de socios por Internet.

Por todo ello, debe concluirse que las ONGs españolas no ofrecen los sistemas de retroalimentación comunicativa mínimos para establecer una relación adecuada con sus públicos, salvo en el caso de admitir sus aportaciones económicas, hecho en el que sí manifiestan un marcado interés y disponen la tecnología para su logro. 


\section{Discusión de resultados}

A nivel expositivo la parcialmente adecuada gestión de la comunicación parece reservada a organizaciones de elevado presupuesto. En nuestra opinión supone un error en el caso de organizaciones de escasa capacidad, ya que es posible implementar herramientas y gestionar contenidos comunicativos a muy bajo coste, como los referidos a la información corporativa que gocen de cierto inmovilismo. Sin embargo, la actualización de noticias necesita de la dedicación de recursos específicos a tal menester, lo cual parece reservado a mayores presupuestos, pues parece poco alcanzable para al menos las ONGs con presupuestos más ajustados.

Por ello, las organizaciones de escaso presupuesto deberían incluir en sus páginas web contenidos más o menos perdurables - por ejemplo, información de tipo corporativo, o información sobre los proyectos más importantes-e implementar herramientas de bajo coste -por ejemplo, buscadores de información-que optimizarían su potencial comunicativo.

En el caso de las organizaciones con elevados presupuestos, se deberían desarrollar con mayor intensidad contenidos con vídeo y audio, ya que fomentan la emotividad y facilitan el desarrollo del discurso comunicativo.

Sin embargo, a nivel retroalimentativo, la tendencia es común: en el sector de las ONGs se desaprovecha totalmente el potencial de este medio, sea cual fuere su presupuesto.

Por ello, y más aún teniendo en cuenta el bajo coste general de implantación de esta tecnología, las ONGs deberían implementarla independientemente del presupuesto manejado. Deberían por tanto tener presencia en redes sociales, disponer de sindicación de contenidos por RSS, usar blogs y mantenerlos periódicamente y permitir al menos el comentario y valoración de lo que publican.

Dicha actuación redundará en la potenciación de bidireccionalidad comunicativa, y en definitiva, tenderá hacia la simetría comunicativa entre la organización y sus públicos, tan importante en organizaciones de este tipo.

Por otra parte, las limitaciones más importantes de este estudio se relacionan con la potencial falta de representatividad del mismo, ya que no hay datos fiables sobre la población objeto de estudio, lo cual impide concretar un grado de error con la exactitud adecuada. Además, en la muestra utilizada (101 organizaciones) se tuvo que renunciar a la proporcionalidad en los estratos, ya que como se ha mencionado anteriormente uno de los objetivos esenciales de esta investigación trataba de determinar diferencias relevantes entre ONGs de distinto presupuesto. 
Futuras líneas de investigación interesantes podrían incluir el estudio de las ONGs en función de su estado comunicativo, y de acuerdo con la teoría de modelos de actuación de Grunig y Hunt (Grunig y Hunt, 2003:72-104). También podría ser de interés el estudio de la comunicación de estas organizaciones desde una perspectiva diferente a Internet, usando diferentes medios y canales que podrían ofrecer resultados alternativos.

\section{Referencias bibliográficas}

Clown K. y Carter E. (1999): "Advertising by non-profit organizations", Proceedings of the Academy of Marketing Studies, Vol. $4, \mathrm{n}^{\circ} 2$.

Diario Digital 20 Minutos (1997): “Detenido el presidente de la ONG Anesvad por apropiación indebida de fondos”, 09-032007. [www.20minutos.es]

Diario Digital 20 Minutos (1997): "Anticorrupción investiga a Intervida por el posible de desvío de fondos de apadrinamientos para fines privados”, 03-04-2007. [www.20minutos.es]

Galán, L. (2005): “La otra cara de las ONG”. Diario El País, edición digital. 05/12/2005.[http://www.elpais.com/articulo/espana/cara/ONG/elpporesp/20051205elpepinac_1/Tes]

Galve Rodríguez, M. M., Caba Pérez, M. C. y López Godoy, M. (2009): “Internet como herramienta clave para la transparencia de las ONG", XV Congreso AECA.

García, A. (2010): “Donar con los ojos abiertos”. Diario Cinco Días, edición digital. 27/09/2010. [Disponible en http://www.cincodias.com/articulo/Sentidos/Donar-ojos-abiertos/20070419cdscdicst_1/cds5se/]

Grunig, J. E. y Hunt, T. (2003): Dirección de relaciones Públicas. Barcelona: Gestión 2000.

Kang S., Norton H. E. (2004): “Nonprofit organizations' use of the World Wide Web: are they sufficiently fulfilling organizational goals?” Public Relations Review, no 30, 2004, pp. 279-284.

Ozcelik, Y. (2008): "Globalization and the Internet: Digitizing the Nonprofit Sector", Journal of Global Business Issues, Vol. 2, $\mathrm{n}^{\circ} 1,2008$.

Peñalva, J. (2004): “De la comunicación tradicional a la comunicación digital: oportunidades y amenazas", en Bel Mallén, J. I. (eds): Comunicar para crear valor. La dirección de comunicación en las organizaciones. Barañáin: Ediciones Universidad de Navarra.

Pérez V. y Mateos C. (2006): “ONG, Internet y Comunicación Alternativa en las ONG dedicadas a la inmigración en España”, Razón y Palabra, nº. 29, p. 49.

Pérez Díaz, V. M. y López Novo, J. P. (2003): El tercer sector social en España. Madrid: Ministerio de Trabajo y Asuntos Sociales. 
Rosenfeld, L. y Morville, P. (2006): Information Architecture for World Wide Web. Designing Large-Scale Web Sites., Sebastopol: Ed. O’Reilly.

Salvador i Peris, P. (2000): Comunicación e imagen en las ONG. Valencia: Servei de Publicacions de la Universitat Jaume I http://www.uji.es/bin/publ/edicions/jfi5/ong.pdf

Sanz de la Tajada, L. A. (1994): Integración de la identidad y la imagen de la empresa, Madrid: ESIC.

Soria Ibáñez, M. M. (2009): “Cómo las ONGs españolas afrontan una crisis de imagen a través de la sala de prensa virtual”, Razón y Palabra, nº 70.

(2009b): “Comunicación 2.0 y salas de prensa virtuales en las organizaciones no lucrativas españolas" RRPP. Net, portal de Relaciones Públicas http://www.rrppnet.com.ar/comunicacion2.0.htm.

Tapia, A., Gómez, B., Herranz, J. M. y Matellanes, M. (2009): “La gestión de la comunicación de las organizaciones de economía social a través de sus portales web”. XII Jornadas de Investigadores en Economía Social. CIRIEC España.

Van Riel, Cees B. M. (2000): Comunicación corporativa, Madrid: Prentice Hall Pearson Educación.

Vidal, V. (2004): “La comunicación en las organizaciones no lucrativas”, en Bel Mallén, J. I. (eds): Comunicar para crear valor. La dirección de comunicación en las organizaciones, Barañáin: Ediciones Universidad de Navarra. 